\title{
Numerical cam profile design with tappet constant speed section in internal combustion engine valve gear and fuel system
}

\author{
Alexander Vasilyev $^{1}$, and Yulia Bakhracheva, ${ }^{2, *}$ \\ ${ }^{1}$ Department of heat engineering and hydraulics, Volgograd State Technical University, 400005 \\ Volgograd p. Lenina 28, Russian Federation \\ ${ }^{2}$ Department of Information Security, Institute of priority technologies, Volgograd State University, \\ 400062 Volgograd p. Universitetsky 100, Russian Federation
}

\begin{abstract}
In modern internal combustion engines the drive of valves of the mechanism of gas distribution and a plunger of the fuel pump of high pressure is carried out by the cam mechanism. The functional requirement imposed to gas distribution mechanism cams is receiving the greatest valve "time-section" when ensuring working capacity and reliability of the valvate drive. One of the main directions of improvement of fuel pumps of high pressure of diesel engines is increase in the maximum pressure of injection. In this work it is offered to improve a numerical method by imposition of the restriction on the first derivative of movement of the pusher on a cam angle of rotation, that is a pusher speed analog. This restriction allows to receive a cam with the site of constant maximum speed of the pusher. It allows to increase the maximum pressure of injection.
\end{abstract}

\section{Introduction}

In modern internal combustion engines, the drive of the valves of the gas distribution mechanism and the plunger of the high-pressure fuel pump is carried out by a cam mechanism. Due to the fact that the high-pressure fuel pump operates under significant loading, the cam profile is subject to strict requirements. The functional requirement for the cams of the gas distribution mechanism is to obtain the greatest" time-section" of the valve while ensuring the efficiency and reliability of the valve actuator. One of the main directions of improvement of fuel pumps of diesel engines is to increase the maximum injection pressure.

There are many methods of profiling of cams of the mechanism of gas distribution and fuel pumps of high pressure, each of which has a number of advantages and disadvantages $[1,2]$. In this paper we consider a numerical method for the synthesis of the law of motion of the pusher $[3,4]$, which allows on the basis of a single approach to form the profile of the cam in accordance with the requirements. It is based on the principle of step-by-step formation of the law of motion of the pusher, taking into account the specified restrictions,

*Corresponding author: bakhracheva@volsu.ru 
which allows to abandon the pre-defined schemes of describing the characteristics of the pusher movement or the configuration of the cam profile. In this case, instead of the analytical law of motion, its numerical representation is used, and the algorithm for the formation of areas of positive and negative acceleration provides the characteristics of the maximum efficiency when performing a set of restrictions on the contact voltage, pressure angle, hydrodynamic lubrication conditions in the cam-pusher interface, the radius of curvature of the cam profile, the reserve factor of the valve springs, as well as the highest derivatives of the pusher movement along the cam rotation angle.

\section{Mathematical model}

In connection with the above, in this paper it is proposed to improve the numerical method by introducing a restriction on the first derivative of the pusher movement along the angle of rotation of the cam, that is, the analog of the speed of the pusher. This restriction allows you to obtain a cam with a portion of the constant maximum speed of the pusher, where the value of the pressure angle is limited.

This method of cam profiling with a constant maximum pusher speed section can also be used in high-pressure fuel pumps. The main task here is to ensure intensive fuel injection. This problem can be solved by increasing the speed of the plunger by changing the cam profile at a constant speed of rotation of the pump shaft. This increases the injection pressure at a constant cyclic supply [2]. In high-pressure fuel pumps, a cam with a section of constant maximum speed makes it possible to obtain such a law of motion of the pusher, at which the speed of movement of the plunger is maximum at the injection site from the geometric beginning to the geometric end. This increases the maximum injection pressure.

It is important to note that along with the introduction of a restriction on the first derivative of the pusher movement in the improved numerical method, there is still a set of restrictions related to ensuring the operability and reliability of the gas distribution mechanism, for example, restrictions on contact voltages in the cam-pusher pair. It is also provided for the calculation of the data necessary for the manufacture and control of the cam profile using various kinematic schemes.

Consider the algorithm of formation of the law of motion of the pusher with the introduction of restrictions on the first derivative of the movement of the pusher. Sections $\mathrm{AB}_{+}$positive and $\mathrm{CB}$ _ negative values of the second derivative are formed respectively from points $\mathrm{A}$ and $\mathrm{C}$ (Fig.1 a, b), in which the initial conditions are set. Plots are formed simultaneously step by step at the equality of values $\mathrm{S}^{\prime}$ at the current points $\mathrm{B}_{+}$and $\mathrm{B}_{-}$with the required degree of accuracy determined by the step $\Delta \varphi$, which can be selected sufficiently small. The value of $\mathrm{S}^{\prime \prime}$ at each step is chosen as the maximum modulo allowed restrictions on derivatives of the third and higher orders calculated by the formula

$$
S_{i}^{(k)}=\frac{S_{i}^{(k-1)}-S_{i-1}^{(k-1)}}{\varphi_{i}-\varphi_{i-1}}
$$

where $S_{i}^{(\mathrm{k})}$ is derivative of $k$-th $\operatorname{order}(k>2)$ in the $i$-th point of intersection of segments; $S_{i}^{(\mathrm{k}-}$ 1) and $S_{i-1}(\mathrm{k}-1)$ - derivatives $(k-1)$-th order, respectively, in the $i$-th and $(i-1)$-th points of intersection of segments; $\varphi_{\mathrm{i}}$ and $\varphi_{\mathrm{i}-1}$ - are angles of rotation of the cam, respectively in the $i$-th and (i-1)-th points of intersection of the segments.

The values of $S$ and $S$ at the current point are determined by numerical integration: 


$$
\begin{aligned}
& S_{i}^{\prime}=S_{i-1}^{\prime}+\frac{\varphi_{i}-\varphi_{i-1}}{2} \cdot\left(S_{i}^{\prime \prime}+S_{i-1}^{\prime \prime}\right) \\
& S_{i}=S_{i-1}+\frac{\varphi_{i}-\varphi_{i-1}}{2} \cdot\left(S_{i}^{\prime}+S_{i-1}^{\prime}\right)
\end{aligned}
$$

where $S^{\prime \prime}, S^{\prime \prime}{ }_{\mathrm{i}-1}$ - values of the second derivative in the $i$-th and $(i-1)$ th point of $S_{i}, S_{i-1}-$ the value of the displacement of the follower in the $i$-th and $(i-1)$-th point.

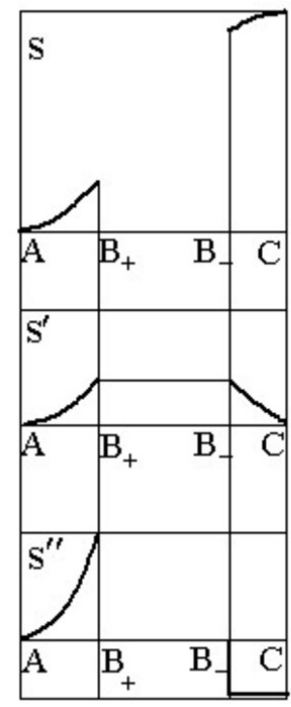

$a$

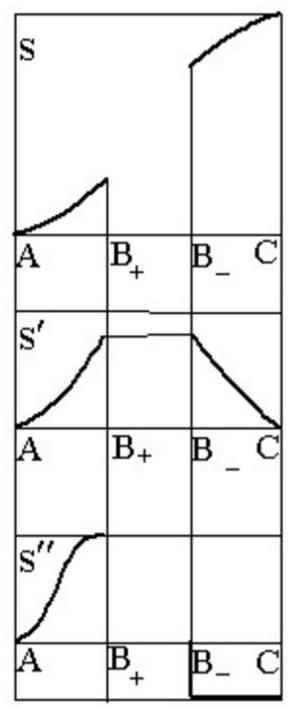

$b$

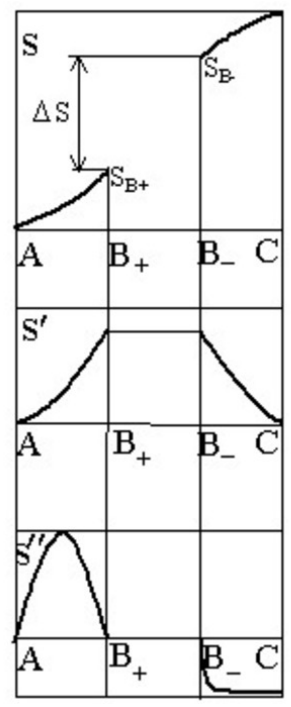

$c$

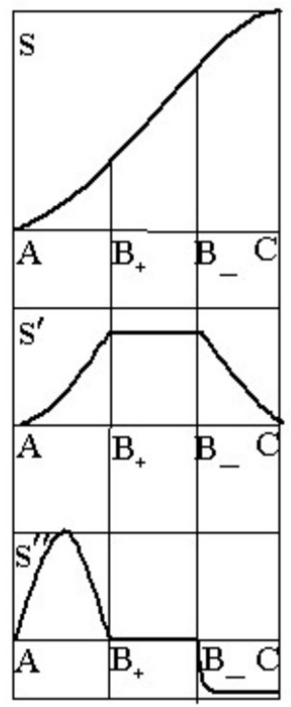

$d$

Fig. 1. Calculation scheme of the formation of the law of motion of the pusher.

Thus, knowing at each step the values of acceleration, speed and displacement of the pusher, we determine by known dependencies [1] the current values of the limited parameters associated with the performance and reliability of the gas distribution mechanism.

If at the current step it is impossible to satisfy the set of restrictions, the module decreases the value of $S^{\prime \prime}$ at one of the previous steps nearest to the current one, and this reduction should be allowed by the existing restrictions (1) on derivatives. Then, the possibility of forming the current step is checked again, and this procedure is repeated until the value of S" satisfying the restrictions is selected at the current step.

In Fig. $1 \mathrm{~b}$, it is seen that with the active restriction on the first derivative of the pusher movement at a certain stage of the formation of the law of motion of the pusher is reduced to zero modules values $S^{\prime \prime}$ at points $\mathrm{b}+$ and $\mathrm{B}_{-}$. The values of $S^{\prime}$ at points b+ and $\mathrm{B}$ are equal to the maximum permissible value of $S$ 'max with the required accuracy determined by the values $\Delta \varphi$ and the correction step $\Delta S^{\prime \prime}$, and the value $\mathrm{s}$ differs by the value $\Delta S$ difference between the values of the pusher movement at points $\mathrm{B}_{-}$and $\mathrm{b}_{+}+$, that is. In figure $1 \mathrm{~b}$, it is seen that with the active restriction on the first derivative of the pusher movement at a certain stage of the formation of the law of motion of the pusher is reduced to zero modules values $S^{\prime \prime}$ at points $\mathrm{B}_{+}$and $\mathrm{B}_{-}$. The values of $S^{\prime}$ at points $\mathrm{B}_{+}$and $\mathrm{B}_{-}$are equal to the maximum permissible value of $S_{\max }$ with the required accuracy determined by the values 
$\Delta \varphi$ and the correction step $\Delta S^{\prime \prime}$, and the value s differs by the value $\Delta S$ difference between the values of the pusher movement at points $\mathrm{B}_{+}$and $\mathrm{B}_{-}$, that is

$$
\Delta S=S_{\hat{A}-}-S_{\hat{A}+}
$$

where $S_{\mathrm{B}-}, S_{\mathrm{B}+}-$ displacements of the follower in points B_and $\mathrm{B}_{+}$(Fig. 1, c) defined by the formula (3).

Formed by a section at constant maximum speed, where the first derivative of the displacement of the pusher according to the rotation angle of the cam has the maximum value, and the value of the displacement of the follower varies linearly (figure $1 \mathrm{~d}$ ).

The length of this section of the constant maximum speed is determined by the formula

$$
\varphi_{0}=\frac{\Delta S}{S_{\text {max }}^{\prime}}
$$

If the restriction on the first derivative of the pusher movement is not active, the formation of the cam profile is performed according to the previously described algorithm $[3,4]$.

Thus, the implemented method of forming the law of motion of the pusher, differs in that the values of the second derivative at the junction points of adjacent segments modulo maximum also when the restriction on the first derivative, and between the sections of positive and negative values can be located a portion of the constant maximum speed, the angular length $\varphi_{0}$ which is determined by the formula (5).

\section{Discussion}

Consider the results obtained in the formation of the law of motion of the fuel pump pusher high pressure. Cam profiling was carried out at the following values of parameters: radius of starting circle of the cam $10 \mathrm{~mm}$, the radius of the cylindrical surface of the follower contacting with the cam, $8.25 \mathrm{~mm}$, the maximum displacement of the follower $8 \mathrm{~mm}$. The specified angular length of the section of the ascent was $63^{\circ}$ angle of rotation of the cam. The limit value can be selected based on the required maximum fuel injection pressure, the limit on the first derivative of the pusher movement was adopted $S_{\max }^{\prime} \leq 7.6 \mathrm{~mm} / \mathrm{rad}$.

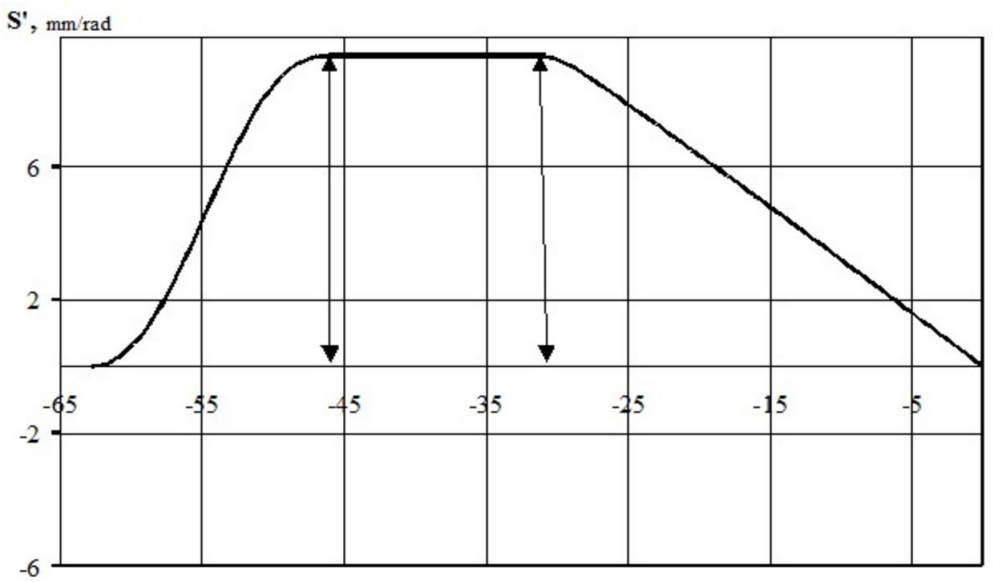

Fig. 2. The speed of movement of the pusher of the fuel pump depending on the angle of rotation of the cam. 
On Fig. 2 the dependence of speed of movement of the pusher of the fuel pump of high pressure depending on a cam angle of rotation is given. Parameters of a profile can be chosen so that points of the geometrical beginning and the geometrical end were on the site of constant maximum speed. It allows to increase the maximum pressure of injection of fuel.

\section{Conclusion}

Thus, the analysis of the obtained results allows us to conclude that the cam with a section of constant maximum speed, profiled by an improved numerical method, can give a number of advantages. Meeting the requirements for the overall dimensions of the plate progressively moving flat pusher can be achieved by limiting the first derivative of its movement. At the same time, the maximum pressure angle in the cam-pusher interface decreases with the tightening of the restriction. It should be noted that in a high-pressure fuel pump, a cam with a constant maximum speed section allows to increase the maximum injection pressure due to the fact that the speed of the plunger movement is maximum at the discharge section.

\section{References}

1. Ju. Bakhracheva, IJCCSE, 9, 21 (2013)

2. A. Baron, J. Bakhracheva, Mechanika, 66, 31 (2007)

3. Yu. Bakhracheva, Science Journal of Volgograd State University. Technology and Innovations, 11, 27 (2014) doi: 10.15688/jvolsu10.2014.2.4

4. Y. Bakhracheva, A. Vasilyev, T. Petikova, J. Materials science forum, 870, 545 (2016) doi: 10.4028/www.scientific.net/MSF.870.545

5. A. Baron, J. Bakhracheva, A. Osipenko, Mechanika, 67, 33 (2007)

6. A. V. Vasilyev, Y. S. Bakhracheva, O. Kabore, Y. O. Zelenskiy, Vestnik Volgogradskogo gosudarstvennogo universiteta, Innovatsionnaya deyatelnost [Science Journal of Volgograd State University. Innovation Activity], 10, 26 (2014)

7. A. V. Vasilyev, Y. S. Bakhracheva, S. Y. Storojakov, Procedia engineering, 150, 312 (2016) doi:10.1016/j.proeng.2016.07.017

8. A. Vasilyev, Y. Bakhracheva, IOP Conf Series: Journal of Phisics: Conf. Series, 1050, 012094 (2018) doi: 10.1088/1742-6596/1050/1/012094

9. Y. Bakhracheva, IOP Conf Series: Journal of Phisics: Conf. Series, 1050, 012006 (2018) doi.org/10.1088/1742-6596/1050/1/012006 